\title{
Taller psicopedagógico para crisis de ansiedad y agarofobia
}

RESUMEN: Estructura y contenidos de un taller psicopedagógico para pacientes con crisis de ansiedad, con o sin agorafobia y sus familiares. Altamente estructurado, con recurso a diverso material de apoyo, durante 1 mes con 4 sesiones de 1,5 horas, con diferentes actividades y "tareas para casa".

Además, se presenta la evolución clínica de 55 participantes, con una significativa reducción en las crisis referidas y niveles de ansiedad generalizada. Junto a ello, un cuestionario de satisfacción recoge una positiva valoración de los participantes.

PALABRAS CLAVE: Crisis de ansiedad, ataque de pánico, agorafobia, grupo psicopedagógico.

\begin{abstract}
Structure and contents of a psychopedagogic group for patients with panic attacks, with or without agoraphobia, and their relatives. Highly structured and using diverse helping materials, runs for 1 month in 4 sessions of 1,5 hours, with different activities and "home work".

It is also presented the clinical evolution of 55 participants, having observed a significant reduction in the number of reported crisis and level of generalised anxiety. With a satisfaction questionnaire, a positive valorisation is elicited from the participants.
\end{abstract}

KEY WORDS: Panic Attack, Agoraphobia, psychopedagogic group.

\section{A.- Introducción}

Las crisis de angustia, por sí solas o asociadas a otras condiciones mentales, son motivo de demanda de atención frecuente en nuestros servicios. Esta patología genera un importante sufrimiento en quien la padece y sus allegados, con una considerable alteración en sus rutinas y hábitos diarios. Además, condicionan una importante disminución del rendimiento laboral y aumento de las bajas laborales. El gasto sanitario generado por estos procesos muy posiblemente no venga dado en su mayor parte por el tratamiento específico del problema, sino por la multiplicidad de exploraciones a que se ven sometidos por sus quejas somáticas.(1-3)

En estos momentos, dado el desbordamiento de los servicios públicos, la mayor parte de los casos se tratan farmacológicamente sin recurso significativo a otras técnicas terapéuticas coadyuvantes. A pesar de estar formalmente indicados, en la práctica resulta muy difícil ofertar abordajes psicoterapéuticos más dilatados a todos los pacientes. A pesar de estas limitaciones, es necesario seguir mejorando en lo posible nuestra oferta asistencial. Para ello, proponemos complementar los tratamientos actuales con una intervención puntual de tipo grupal, intensa, activa, de corta duración y en el primer momento de la demanda.

Rev. Asoc. Esp. Neuropsiq., 2001, vol XXI, n. ${ }^{\circ} 78$, pp. 127-137 
Dentro de los diferentes tipos de abordaje grupal en Salud Mental, hemos elegido el enfoque educativo o psicopedagógico. No podemos dejar de recordar que históricamente este enfoque se reconoce como el pionero en el desarrollo de las técnicas grupales, gracias a las experiencias de Pratt(4) con tuberculosos y que pronto fue utilizado en otro tipo de circunstancias, incluso con diferentes posicionamientos teóricos.(5) (6) El objetivo básico de estos abordajes es ayudar a sus componentes a mejorar sus estrategias de manejo de los problemas reales que estén afectando a sus vidas en el momento actual. Las técnicas específicas se centran en la divulgación de información en forma de clases, manuales o videos, además de consejos, ejercicios grupales, estrategias de resolución de problemas, tareas, etc.

Este tipo de técnica ha sido desarrollado sobre todo para pacientes psicóticos(7-10), aunque su utilización no es exclusiva de esta patología, ni incompatible con otras orientaciones teóricas. Se han descrito experiencias, por ejemplo, en la preparación al abordaje grupal psicodinámico en casos de estrés postraumático tras la guerra de Vietnam(11) o para la preparación previa a la psicoterapia en clases sociales culturalmente menos sofisticadas(12), grupos de medicación(13) e incluso en intervenciones con pacientes asmáticos(14) o sobre la capacidad diagnóstica de médicos de atención primaria15.

De todos modos, en general, el enfoque psicopedagógico no es un abordaje extendido en el campo de las "neurosis". En el caso del trastorno de angustia, junto con la farmacología, podría ser un abordaje básico e incluso, en cierto modo, imprescindible(1) (2) (16) para posteriores acercamientos psicoterapéuticos de otro tipo. Por ejemplo, se ha señalado que en pacientes con un predominio del componente biológico de su enfermedad, la insistencia prematura en la comprensión dinámica o en responsabilizarles de sus síntomas no sólo sería inútil, sino que a largo plazo podría ser potencialmente perjudicial, contribuyendo a la pérdida de autoestima y al fortalecimiento de las defensas masoquistas(17).

\section{B.- Objetivos generales del taller}

1.- Mejorar el conocimiento que el paciente tiene de su patología, en la convicción de que este conocimiento le tranquiliza y ayuda a enfrentar mejor su problemática, pudiendo en muchas ocasiones frenar su escalada de búsqueda de ayuda por motivos somáticos.

2.- Confrontarle con la existencia de otras personas con su misma problemática, tranquilizándole ante la evidencia de que a otros les pasa lo mismo, así como mostrarle la poca originalidad de sus propios síntomas.

3.- Dotarle de técnicas con las que él mismo pueda enfrentar la situación y sentirse actor de su propia mejoría.

4.- Prevenir abandonos, potenciando la adhesión al tratamiento que le sea indicado por el profesional que le atienda. 
INFORMES

5.- Alertarle ante posibles riesgos asociados con el mal uso de la medicación o de aquellos otros derivados de conductas tales como el uso de tóxicos o el sedentarismo.

6.- Sensibilizarle ante cualquier otra área disfuncionante de su vida, así como acerca de la importancia de los aspectos relacionales, ya sean familiares o sociales.

7.- Dotarle de los mínimos conocimientos para saber discriminar entre la variedad de tratamientos psicoterapéuticos disponibles, en el caso de que busque este tipo de ayuda en otro lugar.

8.- Sugerir hábitos saludables de vida.

9.- Apoyar al profesional que le atienda durante las primeras semanas del tratamiento, ofreciéndole la posibilidad de que el paciente sea controlado con una cierta frecuencia, manteniendo él la responsabilidad sobre la dirección del caso.

10.- Apoyar e informar a la familia del paciente.

11.- Mejorar los recursos de tratamiento existentes y en definitiva la calidad asistencial.

12.- Disminuir el gasto sanitario derivado de intervenciones médicas y exploraciones complementarias innecesarias.

\section{C.- Diseño general}

Se busca una intervención limitada y condensada en el tiempo, directiva y de carácter psicopedagógico. Con un importante recurso a estrategias cognitivo-conductuales en los planteamientos globales, no por ello se descuidará la necesidad de "hablar" que presentan los pacientes, favoreciendo su participación activa y prestando especial atención a algunos de los que han venido a llamarse factores terapéuticos grupales de tipo inespecífico (interacción, aceptación, guía, universalismo, altruismo, instilación de esperanza, aprendizaje vicario, etc.)(18).

El taller se ha diseñado con una duración de 4 sesiones cerradas, de 90 minutos semanales, a lo largo de 1 mes. El reducido número de sesiones y su limitación temporal permiten la rápida inclusión de nuevos pacientes y de esta forma dar una pronta respuesta tras los primeros momentos de la demanda. Otra posibilidad a ensayar sería una frecuencia quincenal durante 2 meses, lo que permitiría igualmente una rápida inclusión además de un seguimiento inicial algo más prolongado.

Puede ser candidata a incluirse en el taller cualquier persona, independientemente de su diagnóstico principal, que presente crisis de pánico y/o agorafobia como síntoma evidente y amenazante en su estado. Obviamente habráán de ser excluidos aquellos que por sus características de personalidad, momento psicopatológico o dificultad para comprometerse a participar en todas las sesiones, podemos presuponer dificultarán en extremo el buen desarrollo de la experiencia. 
Dependiendo del lugar y profesionales que dirijan la experiencia (mejor dos que uno), la selección podrá hacerse exclusivamente entre los propios pacientes del responsable del taller, o mejor aún ofrecer la participaciónn en él a pacientes de otros compañeros, que mantendrían su responsabilidad clínica sobre el paciente. Otra posibilidad, ensayada por nosotros con buenos resultados en un limitado número de casos, es ofrecer el "taller" directamente a los propios médicos de A.P. como un servicio concreto. Una vez finalizada la experiencia los pacientes les serían devueltos, dejando siempre abierta la posibilidad de la rederivación. De esta forma, pueden ver reforzadas y apoyadas sus intervenciones y animarse a continuar con el tratamiento y seguimiento de algunos de sus pacientes menos complicados.

Los grupos estarán compuestos por 3 a 5 pacientes, de cualquier edad o sexo. Preferiblemente en los primeros momentos tras su diagnóstico clínico, generalmente más necesitados de contención y como mejor manera de evitar en lo posible una evolución tórpida. Igualmente, podrá invitarse a participar a un familiar por paciente, deseablemente la persona de la que el paciente depende mas y sobre la que recaiga mayor peso en la contención y acompañamiento en los momentos difíciles. Un especial cuidado habrá de tenerse en recalcar que sea siempre el mismo familiar el que acuda a todas las sesiones.

\section{Material}

Además de las instalaciones y personal del propio Centro, es necesario utilizar un mínimo material de tipo documental. La documentación entregada a los participantes ha de tener una presentación de suficiente calidad. La calidad resulta de la máxima importancia como factor de imagen del propio Centro, como señalamiento de la importancia de los contenidos, así como para permitir su conservación una vez finalizado el Taller. De igual forma, es necesario contar con un videorreproductor y monitor de TV (retroproyector o proyector de diapositivas en su defecto), así como un ordenador, impresora y fotocopiadora.

\section{E. Contenidos}

Como forma de ampliar en lo posible el relativamente escaso número de sesiones, además de responsabilizar lo máximo posible al paciente en su propia evolución, se recurrirá a tareas para casa y a la cumplimentación de diferentes cuestionarios. La utilización de cuestionarios y escalas tiene la función de reforzar el reconocimiento de los propios síntomas, así como la valoración personal de su evolución en el tiempo. Sin ser un objetivo principal, también podrán tener una función de apoyo clínico al profesional responsable del caso, así como cierta utilidad para eventuales proyectos de investigación. La utilización de estas estrategias (cuestionarios, material impreso, etc.) previsiblemente será bien recibido y valorado por los pacientes al corresponderse con sus propias 
expectativas hacia el servicio en el momento de pedir consulta, tal y como hemos constatado en otro lugar(19).

Un aspecto de gran importancia es el relativo a la información a suministrar al paciente. Esta información es presentada en forma de pequeño libro o "manual", que deseablemente debe ofrecer los máximos contenidos posibles, fiables y en profundidad. Todo ello no sólo como forma de garantizar la mayor cantidad de información en un primer momento, sino como la mejor manera de dejar a disposición del paciente un material que podrá ser consultado en tiempos futuros. En la actualidad ha sido editada en forma de libro (20) para su distribución gratuita entre pacientes de Atención Primaria y Servicios Especializados y que no necesariamente participen en el taller. El texto completo también está disponible a través de Internet(21).

Los contenidos del Taller se estructuran básicamente en dos grandes grupos: "Tareas" y "Sesiones". Las Tareas son aquellas actividades que deberá realizar el paciente individualmente en su domicilio, antes de cada una de las Sesiones que tendrán lugar de forma grupal en el Centro de Salud Mental.

Las Tareas vendrán marcadas y dirigidas a través de la documentación oportuna, entregada en el momento en que se decide su participación en el taller y al final de cada una de las sesiones. A continuación se describen cada una de estas tareas y los contenidos de las sesiones.

Las sesiones se componen de diferentes actividades tales como: visionado de un video, valoración y discusión de los resultados agregados del cuestionario sintomatológico, control y discusión de dudas de los cuestionarios de autoevaluación, discusión de las redacciones, reforzamiento de estrategias personales de afrontamiento, confrontación con los puntos de vista de los familiares, etc.

\section{F. Seguimiento}

Una vez finalizado el taller, la disposición de los pacientes es diversa. Como se ha reflejado anteriormente, en algunos casos concretos se han devuelto para su seguimiento a A.P. Otros reciben nuevas consultas programadas de seguimiento o son incluidos posteriormente en programas de psicoterapia más sofisticados. Sin embargo, para una gran mayoría queda abierta la posibilidad de consultar, personal o telefónicamente, dependiendo de su evolución, habiendo observado que la frecuencia y angustia con las que se solicitan estas consultas han disminuido de forma muy evidente.

En todos los casos, además de reforzar sus capacidades de autocontrol, se recomiendan otros recursos disponibles. El manual juega un papel muy importante en el apoyo posterior, recomendándose volver a leerlo en momentos críticos de rebrotes ansiosos. Igualmente se aconseja la participación en "grupos de autoayuda", recientemente 
INFORMES

desarrollados en nuestro entorno y que pueden jugar un papel muy importante en la evolución de algunos pacientes.

La posibilidad de disponer de gran parte del material del taller en Internet, no sólo ha permitido su consulta a otras personas y familiares afectados, sino que ha beneficiado posteriormente a alguno de los participantes, abriendo de esta forma la posibilidad a unos y otros de ampliar el apoyo gracias a otros recursos específicos del medio ("Chat", grupos de autoayuda a través de foros y correo electrónico, etc.).

Por último, estamos considerando en la actualidad la oportunidad de ampliar el taller con algunas sesiones de "refuerzo", por ejemplo mensualmente durante 6 a 12 meses. Quizás no necesariamente para todos los participantes y con la posibilidad de mezclar 263 grupos con este fin. En este último caso, podría ser interesante reagrupar a los participantes según otros rasgos asociados (agorafobia, hipocondría, somatización, depresión...). Igualmente, puede ser muy interesante seguir explorando las potencialidades de Internet con estos objetivos.

\section{G. Evaluacion de la experiencia}

El primer taller se llevó a cabo a finales de 1996. Hasta el momento se han realizado 12 grupos con 55 participantes en total (Tabla I). No fue nuestra intención, en un principio, la de realizar un diseño experimental que estudiara el impacto del taller sobre la sintomatología de los participantes. Aún así contamos con los cuestionarios clínicos realizados al inicio y final de la experiencia. Sin disponer de un grupo control de características similares y que sin haber pasado por el taller puedan contrapesar el papel jugado por la propia evolución del cuadro o el tratamiento farmacológico coadyuvante, los resultados observados más destacables son:

En los días previos a su incorporación al taller, los participantes refieren ya cierta mejoría con respecto al mes anterior, según reflejan en una escala lineal (del 1 al 60) de "mucho peor" a "mucho mejor". Esta mejoría es referida como aún mayor al término de la experiencia y en este caso en compración con los momentos previos al inicio del taller. Dado que la escala no pregunta sobre la situación en ese momento, sino sobre el grado de mejoría en relación con el mes anterior, no parece oportuno valorar la diferencia directa entre ambos momentos (Tabla II). Esta compración sin es posible, con el número de crisis de ansiedad que aparecen en la semana previa a ambos momentos de la evaluación. Tanto en el caso de las crisis espontáneas como de las situacionales, se observa un importante número de pacientes que ven disminuidas el número de crisis $(\mathrm{P}<0,001)$. De igual forma que refiren una significativa disminución $(P<0,05)$ en los niveles de ansiedad genrealizada (Tabla III).

Por otra parte, al término de la experiencia se entrega un cuestionario solicitando opiniones y sugerencias en relación con el taller (Tabla IV). En relación exclusivamente 


\section{INFORMES}

con el manual, contamos además con la opinión de varios cientos de personas que respondieron de forma anónima a través de Internet un cuestionario diseñado con este fin, refiriendo casi únanimemente el interés por sus contenidos, la importancia de los enfoques informativos para un mejor abordaje de su problemática y la falta de información específica acerca de su trastorno incluso durante años de su tratamiento.

Desde el punto de vista asistencial, y sin poder presentar resultados "objetivos", las opiniones recibidas desde A.P. son alentadoras. Además, tenemos la sensación subjetiva de contar con una nueva herramienta de abordaje en casos "difíciles", por su tendencia a la búsqueda de explicaciones somáticas, que ha permitido contener y distanciar parte de esta demanda.

\begin{tabular}{|l|l|l|l|}
\hline & Hombres & Mujeres & Total \\
\hline Sexo & 20 & 35 & 55 \\
Edad media & 36.35 & 31.23 & 33.09 \\
E. civil & & & \\
Soltero & 8 & 15 & 23 \\
Casado & 11 & 17 & 28 \\
Sep-Divorciado & 1 & 2 & 3 \\
Viudo & -- & 1 & 1 \\
Nivel Instrucción & & & \\
Primarios - Elemental & 13 & 19 & 32 \\
Bach. Superior & 5 & 12 & 17 \\
E. Medios - Superiores & 2 & 4 & 6 \\
Laboral & & & \\
Activos & 9 & 14 & 23 \\
Paro & 7 & 4 & 11 \\
Pens. Incapacitado & 2 & 1 & 3 \\
Estudiante & 1 & 6 & 7 \\
Ama de Casa & -- & 10 & 10 \\
\hline
\end{tabular}

TABLA I. Características sociodemográficas básicas de los participantes 


\begin{tabular}{|c|c|c|}
\hline \multicolumn{2}{|c}{} & $\begin{array}{c}\text { ¿Cómo estaba } \\
\text { hace 1 Mes? }\end{array}$ \\
\hline & $\begin{array}{c}\text { Respuesta } \\
\text { antes del Taller }\end{array}$ & $\begin{array}{c}\text { Respuesta } \\
\text { después del Taller }\end{array}$ \\
\hline Hombres (15) & 37.33 & 43.93 \\
Mujeres (30) & 40.49 & 45.03 \\
Total (45) & 39.38 & 44.67 \\
\hline
\end{tabular}

TABLA II. Valoración subjetiva del estado general de la persona, comparativamente con su estado un mes antes.

\begin{tabular}{|l|c|c|c|}
\hline $\begin{array}{l}\text { Diferencia entre antes } \\
\text { y después del taller }\end{array}$ & $\begin{array}{c}\text { Crisis } \\
\text { Espontáneas }\end{array}$ & $\begin{array}{c}\text { Crisis } \\
\text { situacionales }\end{array}$ & $\begin{array}{c}\text { Ansiedad } \\
\text { generalizada }\end{array}$ \\
\hline $\begin{array}{l}\text { Casos con menos crisis } \\
\text { onivel de ansiedad }\end{array}$ & 24 & 22 & 20 \\
\hline $\begin{array}{l}\text { Casos con más crisis } \\
\text { o nivel de ansiedad }\end{array}$ & 1 & 2 & 6 \\
\hline $\begin{array}{l}\text { Casos con igual no crisis } \\
\text { o nivel de ansiedad }\end{array}$ & 19 & 20 & 19 \\
\hline $\begin{array}{l}\text { P(Test de Wilkonson para muestras } \\
\text { emparejadas) }\end{array}$ & $<0.001$ & $<0.001$ & $<0.05$ \\
\hline
\end{tabular}

TABLA III. Comparación entre el número de crisis espontaneas y situacionales, así como los niveles de ansiedad generalizada, en la semana previa al inicio y finalización del taller. 


\begin{tabular}{|c|c|c|}
\hline & $\begin{array}{l}\text { Pacientes } \\
\text { (46) }\end{array}$ & $\begin{array}{l}\text { Familiares } \\
\text { (20) }\end{array}$ \\
\hline $\begin{array}{l}\text { Interés General } \\
\text { Sin mínimo interés }(0) \text { - Indiferente (5/6) } \\
\text { Muy interesante (10) }\end{array}$ & 8.86 & 8.62 \\
\hline $\begin{array}{l}\text { Utilidad General } \\
\text { Ha complicado más las cosas (0) - Indiferente (5/6) } \\
\text { Muchísima (10) }\end{array}$ & 8.00 & 8.00 \\
\hline $\begin{array}{l}\text { Duración } \\
\text { Muy insuficiente (0) - Suficiente, pero condensado (5/6) } \\
\text { Excesivo (10) }\end{array}$ & 6.14 & 5.57 \\
\hline $\begin{array}{l}\text { Cuestionarios } \\
\text { Muy mal (0) - Regular (5/6) - Muy bien (10) }\end{array}$ & 8.33 & 8.00 \\
\hline $\begin{array}{l}\text { Manual } \\
\text { Muy mal (0) - Regular (5/6) - Muy bien (10) }\end{array}$ & 8.73 & 8.58 \\
\hline $\begin{array}{l}\text { Extensión del Manual } \\
\text { Poca extensión (0) - Adecuada (5/6) - Demasiado extenso (10) }\end{array}$ & 5.98 & 5.57 \\
\hline $\begin{array}{l}\text { Profundidad de Contenidos del Manual } \\
\text { Demasiado superficial (0) - Adecuada (5/6) } \\
\text { Demasiado profundo (10) }\end{array}$ & 6.38 & 6.43 \\
\hline $\begin{array}{l}\text { Claridad de Redacción del Manual } \\
\text { Demasiado simple o coloquial (0) - Adecuada (5/6) } \\
\text { Poco Claro (10) }\end{array}$ & 6.49 & 6.07 \\
\hline $\begin{array}{l}\text { Videos } \\
\text { Muy mal (0) - Regular (5/6) - Muy bien (10) }\end{array}$ & 7.90 & 7.80 \\
\hline $\begin{array}{l}\text { Redacciones Personales } \\
\text { Muy mal (0) - Regular (5/6) - Muy bien (10) }\end{array}$ & 7.72 & 8.24 \\
\hline $\begin{array}{l}\text { Discusiones Grupales } \\
\text { Muy mal (0) - Regular (5/6) - Muy bien (10) }\end{array}$ & 8.27 & 8.25 \\
\hline $\begin{array}{l}\text { Posibilidad de acudir familiares } \\
\text { Muy mal (0) - Regular (5/6) - Muy bien (10) }\end{array}$ & 8.67 & 9.05 \\
\hline $\begin{array}{l}\text { Listado de síntomas de los participantes } \\
\text { Muy mal (0) - Regular (5/6) - Muy bien (10) }\end{array}$ & 7.74 & 7.65 \\
\hline
\end{tabular}

TABLA IV. Valoración, por pacientes y familiares, de diferentes aspectos del taller en una escala del 1 al 10. 


\section{BIBLIOGRAFIA:}

1. Hollander, E. Simeon, D. Gorman, J.M. "Trastornos de ansiedad". En Hales, R.E. Yudofsky, S.C. Talbott, J.A. (Ed.) Tratado de Psiquiatría $2^{a} E d$. The American Psychiatric Press, Ancora. Barcelona, 523- 595, 1996.

2. Kaplan, H.I. Sadock, B.J. Grebb, J.A. Sinopsis de Psiquiatría. Ed. Médica Panamericana. Bogotá. 1996.

3. Ros, S. Lupresti, C.E. Pánico-Agorafobia. Ed. Arán. Madrid. 1999.

4. Pratt,J.H. "The tuberculosis class : an experiment in home treatment (1917)" Reimpreso en Rosenbaum, M. Berger, M. (Ed.) Group Psychotherapy and Group Function. Basic Books, New York. Edición revisada. 1975, 131-142.

5. Lazell, E.W. "The group treatment of dementia praecox". Psychoanal. Rew. 8: 168,1921 .

6. Marsh, I.C. "An experiment in the group treatment of patients at the Worcester State Hospital". Ment. Hyg. 17: 397, 1933.

7. Fenn, H.H. Dinaburg, D. "Didactic group psychotherapy with chronic schizophrenics". Int. J. Group Psychother. 31: 443, 1981.

8. Liberman, R.P. "Psychosocial treatments for schizophrenia" Psychiatry 57(2) : 104-14, 1994.

9. Kissling, W. "Compliance, quality assurance and standards for relapse prevention in schizophrenia". Acta Psychiat. Scand. 89 (s. 382) : 16-24, 1994.

10. Yáñez, R. Carbonell, C. Montañés, F. "Programas psicoeducativos, emoción expresada y calidad de vida en la esquizofrenia". Anales de psiquiatría 15 : 1-7, 1999.

11. Koller, P. Marmar, C.R. Kanas, N. "Psychodinamic group treatment of post-traumatic stress disorder in Vietnam veterans". Int. J. Group Psychother. 42: 225, 1992.

12. Jones, E. Matsumoto, D.R. "Psychotherapy with the underserved". En Snowden, L.R. (Ed.) Reaching the underserved: Mental health needs of neglected populations. Beverly Hills. Sage, 207-228, 1982.

13. Brook, D.W. "Medication groups". En Alonso, A. Swiller H.I. (Eds.) Group Psychotherapy in Clinical Practice. American Psychiatric Press, Washington, 1992.

14. Sommaruga, M. Spanevello, A. Migliori, G.B. et al. "The effects of a cognitive behavioural intervention in asthmatic patients" Monaldi Arch. Chest. Dis. 50(5) : 398-402, 1995.

15. Andersen, S.M. Harthorn, B.H. "Changing the psychiatric knowledge of primary care physicians. The effect of a brief intervention on clinical diagnosis and treatment". Gen. Hosp. Psychiatry. 12(3) : 177-90, 1990.

16. Brook,D.W. "Terapia de grupo en la ansiedad y trastornos del humor". En Kaplan, H.I. Sadock, B.J. (Eds.) Terapia de Grupo. $3^{a}$ Edición. Ed. Méédica Panamericana, Madrid. 1996. 
INFORMES

17. Cooper, A.M. "Will neurobiology influence psychoanalysis?". Am. J. Psychiatry $142: 1395-1402,1985$.

18. Bloch, S. Crouch, E. Therapeutic Factors in Group Psychotherapy. Oxford University Press. Oxford, 1985.

19. Martínez Azumendi, O. Beitia, M. Araluce, K.. et al. "Estudio de las primeras consultas en un Centro de Salud Mental. (II) Características clínicas, sucesos vitales, ajuste y apoyo social, motivación y expectativas". Rev. Asoc. Esp. Neuropsiquiatría. 1997 ; XVII(62) : 203-222.

20. Martínez Azumendi, O. Crisis de Pánico y Trastorno de Angustia. Manual para el Paciente. OME - Asoc. Esp. Neuropsiquiatría - SmithKline Beecham. Madrid, 1999.

21. Martínez Azumendi, O. La Crisis de Ansiedad, Pánico o Angustia. [Publicación “online"]. Disponible en URL: http//www.geocities.com/HotSprings/6333

$\left(^{*}\right)$ Psiquiatra

CENTRO DE SALUD MENTAL DE URIBE COSTA

OSAKIDETZA - SERVICIO VASCO DE SALUD.

SAN NICOLAS 2

48990 GETXO - BIZKAIA

e-mail: oscarmar @ intersalud.es

Fecha de recepción: 5-6-2000 